\title{
Herramientas ofimáticas aplicadas en los procesos administrativos en las instituciones del cantón Salcedo y Pujilí.
}

\author{
Office automation tools applied in administrative processes in the \\ institutions of the canton Salcedo and Pujili
}

\author{
Galo Alfredo Flores Lagla. ${ }^{1}$, Diego Fernando Jácome Segovia. ${ }^{2}$, Lorena Maricela \\ Paucar Coque. ${ }^{3} \&$ Yadira Paola Borja Brazales. ${ }^{4}$
}

Recibido: 09-07-2021 / Revisado: 23-07-2021 /Aceptado: 06-08-2021/ Publicado: 05-09-2021

\begin{abstract}
DOI: https://doi.org/10.33262/concienciadigital.v4i3.2.1844

Introduction: The office applications used in administrative processes is the fundamental element in document management. The research allowed to identify the different types of office tools that are used in the management, administration and management of information in the institutions of the canton Salcedo and Pujilí, of the province of Cotopaxi. The research approach is quantitative, descriptive level and cross-sectional, as a technique a survey validated by experts in the areas of interest was applied. The population considered for the study corresponded to the administrative staff of the three public institutions and one private one. Among the results, it is highlighted that female staff is the predominant axis; The activities they carry out are the following: document management, handling of physical and digital information, archival and customer service. Microsoft Word is the word processor used in these institutions with $87.50 \%$ acceptance. The most used office applications are Microsoft Word and Excel, the same ones that have become a reference in the 4 institutions due to their multiple benefits. With these office

\footnotetext{
${ }^{1}$ Universidad Técnica de Cotopaxi, galo.flores@utc.edu.ec, https://orcid.org/0000-0002-8774-3581

${ }^{2}$ Universidad Técnica de Cotopaxi, diego.jacome@utc.edu.ec, https://orcid.org/0000-0001-7681-5386

${ }^{3}$ Universidad Técnica de Cotopaxi, lorena.paucar@utc.edu.ec, https://orcid.org/0000-0003-0972-7962

${ }^{4}$ Universidad Técnica de Cotopaxi, yadira.borja@utc.edu.ec, https://orcid.org/0000-0003-2006-9534
} 
applications, activities related to administrative processes, document management and the preparation of physical and digital information are carried out.

Keywords: office automation tools, document management, ICT

\section{Resumen}

Introducción: Las aplicaciones ofimáticas utilizadas en procesos administrativos es el elemento fundamental en la gestión documental. La investigación permitió identificar los diferentes tipos herramientas ofimáticas que se utilizan en el manejo, administración y gestión de la información en las instituciones del cantón Salcedo y Pujilí, de la provincia de Cotopaxi. El enfoque de investigación es de tipo cuantitativo, nivel descriptivo y corte transversal, como técnica se aplicó una encuesta validada por expertos en las áreas de interés. La población considerada para el estudio correspondió al personal administrativo de las tres instituciones públicas y una privada. Entre los resultados se destacan que el personal femenino es el eje predominante; las actividades que cumplen son las siguientes: gestión documental, manejo de información física y digital, archivística y atención al cliente. Microsoft Word es el procesador de texto utilizado en estas instituciones con el 87,50\% de aceptación. Las aplicaciones de oficina más utilizadas son Microsoft Word y Excel, las mismas que se han convertido como referente en las 4 instituciones por sus múltiples beneficios. Con estas aplicaciones de oficina se llevan a cabo actividades relacionadas con los procesos administrativos, gestión documental y la elaboración de información física y digital.

Palabras Clave: herramientas ofimáticas, gestión documental, TIC.

\section{Introducción}

Las TIC (Tecnologías de la Información y Comunicaciones) son las tecnologías que se necesitan para la gestión y transformación de la información, y muy en particular el uso de ordenadores y programas que permiten crear, modificar, almacenar, proteger y recuperar la información (Sánchez, 2008, p. 156). Las TIC han transformado la manera de trabajar y gestionar la información, son un elemento clave para hacer que las actividades sean más productivas: agilizando la comunicación, sustentando el trabajo en equipo, gestionando las existencias, realizando análisis de datos entre otras cosas (Cano, 2018, p.504).

En diciembre de 2019 se originó en China una enfermedad viral por Coronavirus (covid19) que se convirtió en unos meses en pandemia, con efectos devastadores para todos los integrantes de la sociedad (Yi et al., 2020). En el interconectado mundo moderno, la incertidumbre y sorpresa por lo rápido que se ha alterado la vida de prácticamente todos los habitantes del planeta ha creado una situación inédita para todas las organizaciones, incluyendo las instituciones educativas (Sanz, 2020) quienes se han visto obligadas a suspender las actividades de manera presencial y han optado por el teletrabajo. 
La implementación de las TIC ha producido importantes cambios en el sector público y privado; desde la forma de organización personal e institucional hasta el proceso de comunicación y aprendizaje. Esto es el resultado del uso de diversas tecnologías e innovaciones que han permitido implantar de manera exitosa procesos participativos y procedimientos eficientes y transparentes que ofrecen servicios eficaces al ciudadano simplificando los procesos de la Administración y gestión documental, con mejor calidad y menores costes (Martín, 2019).

Según, (Mujica, 2000, como se citó en Cano, 2018, p. 502), considera que el avance tecnológico de la informática, la computación, y las telecomunicaciones, incorporaron en las organizaciones un enfoque diferente al habitual para acceder al conocimiento, flexibilidad, interactividad, economía, rapidez, independencia, comunicación y desarrollo. Las TIC son herramientas imprescindibles para mejorar la productividad en las empresas, la calidad, el control y facilitar la comunicación, entre otros beneficios, aunque su aplicación debe llevarse a cabo de forma inteligente.

Las TIC en el campo empresarial e institucional permite mejorar los procesos y aumentar su productividad, calidad y control; es necesario señalar que el usar las tecnologías de la información y comunicación se garantizará que los procesos administrativos se manejen con eficacia y eficiencia, siempre y cuando el personal esté capacitado en manejar los recurso tecnológicos, como también es de vital importancia poseer un conocimiento de los procesos internos y externos de la organización para determinar las necesidades tecnológicas que presenta la organización, por lo cual es necesario una planificación de los recursos tecnológicos y de esta manera ir implementando los sistemas informáticos según las necesidades que presenta la organización.

La innovación tecnológica (hardware y software) en las organizaciones es fundamental, porque permite automatizar los procesos, garantizar seguridad, manejo de información digital y como resultado se ofrece una mejor atención al cliente. Sin embargo, por razones de costos, en la mayoría de los casos las TIC son solo para un segmento muy particular de organizaciones, es decir, para aquellas que pueden solventar las fuertes inversiones que demandan la adquisición, implementación y mantenimiento o incluso el desarrollo de software específico o hardware especial para la organización.

El software libre (free software) es el que, una vez desarrollado, puede ser usado, copiado, estudiado, modificado y redistribuido libremente. El software libre es propiedad de todos, cada persona en el mundo tiene derecho a usar el software, modificarlo y copiarlo de la misma manera que sus autores (...) no es necesario pagar por su uso (Hernández, 2005, p. 5, como se citó en Valverde, 2007, p. 3). Por ejemplo dentro de los paquetes ofimáticos libres tenemos Libre Office, WPS office, entre otros y dentro de los sistemas operativos libres tenemos Ubuntu, Mandriva entre otros.

Según (Arriola, Tecuatl \& González, 2011) afirman que el software no libre, también llamado software privado o con licencia, se refiere a cualquier programa informático en el que los usuarios tienen limitadas las posibilidades de usarlo, modificarlo o redistribuirlo, cuyo código no esté disponible o el acceso a éste se encuentre restringido. 
Por ejemplo dentro de los paquetes ofimáticos más usados con licencia esta Microsoft Office en sus diferentes versiones y el sistema operativo más usado con licencia es Windows en sus diferentes versiones.

Las herramientas digitales han cambiado de forma irreversible el mundo en que vivimos, generando un resultado positivo, aportando al desarrollo económico favoreciendo al clima organizacional, mejorando la calidad de vida de los servidores públicos y privados; brindando mejor atención al público en general. Hoy en día, las organizaciones necesitan simplificar las labores de oficina para hacer el trabajo más práctico y dinámico. Para lograrlo, implementan herramientas digitales que les permiten automatizar, optimizar y mejorar la operatividad de la organización, a esto se le conoce como ofimática.

Una herramienta ofimática es una recopilación de programas usados en oficinas que permiten crear, modificar, organizar, escanear, imprimir, etc. archivos y documentos. Son ampliamente usados en paquetes, las suites dominantes del mercado es Microsoft Office (software con licencia), Libre Office (software libre) y aplicaciones online como Suite de Google y Office 365.

De acuerdo a la investigación realizada para un mejor análisis a las herramientas ofimáticas se les clasificó en 2 grupos: los procesadores de texto como por ejemplo: Microsoft Word, Abi Word, Google Docs, Open Office Writer, Bloc de notas y las aplicaciones de uso general que se utilizan en una oficina como son: Microsoft Office, Suite de Google, Office 365, navegadores, servicio en la nube, etc.

Una de las mejores novedades de los últimos años son las denominadas herramientas colaborativas, un conjunto de software y aplicaciones Online que permiten trabajar en espacios compartidos en la red con otros compañeros de la empresa, haciendo que la comunicación y la efectividad sean mucho mejor que si trabajamos en modo local.

La Suite de Google es uno de los servicios que ofrece Google. Como la mayoría de sus aplicaciones es completamente gratis; aunque también existe una versión de pago especialmente diseñada para clientes empresariales. Estas aplicaciones Online proporcionan herramientas eficaces para la gestión y personalización de utilidades para dominios o nombres de Internet. Es decir, permite gestionar el correo electrónico a través de Gmail, mensajería instantánea entre miembros de una organización o red a través de Google Talk, calendario en línea para generar reuniones y recordatorios de fechas especiales Google Calendar, edición de Documentos igualmente en línea con Google Docs, entre otras cosas (Ávila, 2011, p. 51).

Los paquetes ofimáticos son aplicaciones informáticas que facilitan, mejoran, optimizan y automatizan las tareas de la oficina. Permite automatizar tareas como elaboración, modificación y compartición de información a través de dispositivos portables, redes LAN o de las nubes informáticas. En la implementación de un sistema de información intervienen muchos factores, siendo uno de los principales el factor humano. Es previsible que ante una situación de cambio el personal se muestre renuente a adoptar los nuevos procedimientos o que los desarrolle plenamente y de acuerdo a los lineamientos que se 
establecieron. Por esta razón es necesario hacer una planeación estratégica tomando en cuenta las necesidades presentes y futuras de la empresa.

A través del presente trabajo investigativo se ha logrado determinar la importancia de la innovación y de la utilización de los recursos tecnológicos en una empresa pública y privada. Sin duda alguna desde el aparecimiento del COVID 19, las organizaciones han tomado la decisión de implementar el uso de aplicaciones de software en las organizaciones mismas que ayudan en los procesos de gestión documental, elaboración y almacenamiento de información física y digital, realizar trabajos colaborativos desde la nube, compartición de información digital, manejo de documentos administrativos, agendar reuniones de negocios, la comunicación interna y externa a través de e_mail. Entre otras cosas.

Se ha demostrado que en estas organizaciones la cultura digital ha mejorado ya que para realizar las diferentes actividades laborales utilizan aplicaciones de software adecuados para el manejo de la información. Con lo expuesto se logró cumplir con el objetivo central de la investigación que fue identificar los diferentes tipos de herramientas ofimáticas aplicadas en los procesos administrativos en las instituciones del cantón Salcedo y Pujilí.

\section{Método}

Para el desarrollo de la investigación, se realizó el levantamiento de la información en 2 instituciones públicas del cantón Salcedo, que corresponden al Gobierno Autónomo Descentralizado [GAD] y al Hospital Básico [Yerovi Mackuart]. En el primer caso participaron estudiantes de segundo ciclo paralelo "A" y "C" de la Carrera de Gestión de la Información Gerencial de la Universidad Técnica de Cotopaxi, durante el período académico octubre 2019 - marzo 2020. Además, se levantó la información en una institución pública del cantón Pujilí que corresponde al Cuerpo de Bomberos y una institución privada que corresponde a la Cooperativa de Ahorro y Crédito Occidental. En esta segunda investigación participaron estudiantes de segundo ciclo paralelo "A" de la Carrera de Gestión de la Información Gerencial de la Universidad Técnica de Cotopaxi, durante el período académico octubre 2020 - marzo 2021; también colaboraron docentes que imparten las asignaturas relacionadas con la cátedra integradora en el mencionado ciclo.

La investigación está desarrollada bajo un enfoque cuantitativo, de nivel descriptivo y corte transversal, como técnica se aplicó la encuesta cuyo instrumento de medición es el cuestionario. La aplicación del mismo instrumento se realizó en 2 períodos académicos octubre 2019 - marzo 2020 y octubre 2020 - marzo 2021, se construyó a partir de los referentes teóricos que corresponden a las TIC y a las aplicaciones de escritorio y oficina que se utiliza para la gestión de la información en el contexto administrativo en ambientes empresariales, con el aporte de los docentes especialistas en las áreas abordadas se estructuró la herramienta de recolección de datos. El cuestionario fue sometido a validación de juicio de expertos y finalmente el instrumento final quedó compuesto por veinte preguntas cerradas. 
La población considerada para el estudio correspondió al personal administrativo que labora en las oficinas de las Instituciones Públicas y Privadas de la provincia de Cotopaxi ubicadas en los cantones de Salcedo y Pujilí con las cuales la Carrera de Gestión de la Información Gerencial de la Universidad Técnica de Cotopaxi mantiene convenios específicos de cooperación para la realización de prácticas pre-profesionales. En estas instituciones, no están consideradas el número total de personal administrativo, en razón de que existe funcionarios que desarrollan trabajos de campo que no necesariamente requieren de una labor de oficina. Además, en el periodo octubre 2020 - marzo 2021 el mundo entero y Ecuador estaba afectado por el COVID-19, muchas instituciones cerraron sus puertas, realizaban teletrabajo por este motivo el número de encuestados en este período es limitado.

En concordancia con lo anterior, en lo que respecta al tipo de muestreo, se aplicó el muestreo no probabilístico intencional o de conveniencia, "Este método trata de conseguir muestras representativas cualitativamente, (...). Es decir, cumplen con características de interés del investigador, además de seleccionar intencionalmente a los individuos de la población a los que generalmente se tiene fácil acceso. (Hernández \& Carpio, 2019, p. 78). En el presente trabajo investigativo, los criterios estuvieron relacionados con la accesibilidad a los funcionarios encuestados, la apertura brindada por las autoridades de las distintas entidades públicas y privadas seleccionadas y la inclusión de los servidores que desarrollaban únicamente labores de oficina, pues son aquellos quienes día a día están en constante interacción con las aplicaciones de oficina que apoyan sus tareas administrativas. En base a lo descrito se detalla a continuación la población y muestra en la siguiente tabla.

Población y Muestra.

Tabla 1

Población encuestada

\begin{tabular}{lcc}
\hline \multicolumn{1}{c}{ Instituciones Públicas y Privadas } & Población encuestada & Porcentaje \\
\hline Gobierno autónomo Descentralizado del cantón Salcedo (GAD-S) & 60 & $56 \%$ \\
Hospital Básico de Salcedo (Yerovi Mackuart) & 34 & $32 \%$ \\
Cuerpo de Bomberos del Cantón Pujilí & 8 & $7 \%$ \\
Cooperativa de Ahorro y Crédito Occidental del cantón Pujilí & 5 & $5 \%$ \\
TOTAL & $\mathbf{1 0 7}$ & $\mathbf{1 0 0 \%}$
\end{tabular}

Fuente: Autores (2021)

\section{Resultados.}

Como parte de los datos obtenidos, dentro del proceso de levantamiento de información contenida en el instrumento diseñado, se obtuvo como primer resultado, la información demográfica que permite contextualizar la población de estudio. 
Datos Demográficos.

Género

Tabla 2

Personal encuestado que tiene a su cargo una computadora identificada por género.

\begin{tabular}{lcccccc}
\hline Género & $\begin{array}{c}\text { GAD } \\
\text { Municipal } \\
\text { de Salcedo }\end{array}$ & $\begin{array}{c}\text { Hospital Básico } \\
\text { Yerovi Mackuart de } \\
\text { Salcedo }\end{array}$ & $\begin{array}{c}\text { Cuerpo de } \\
\text { Bomberos de } \\
\text { Pujilí }\end{array}$ & $\begin{array}{c}\text { Cooperativa de Ahorro y } \\
\text { Crédito Occidental de } \\
\text { Pujilí }\end{array}$ & Total & Porcentaje \\
\hline Femenino & 31 & 22 & 4,0 & 4 & 61 & $57,01 \%$ \\
Masculino & 29 & 12 & 4,0 & 1 & 46 & $42,99 \%$ \\
Total & 60 & 34 & 8 & 5 & 107 & $100,00 \%$
\end{tabular}

Fuente: Autores (2021)

En la Tabla 2 se identifica el número total de encuestados clasificado por género, demostrando que existe mayor porcentaje de mujeres $(57,01)$ ocupando espacios de trabajo que están relacionados con los procesos administrativos y de gestión documental que se desarrollan en las instituciones consideradas en el estudio.

\section{Resultados alcanzados.}

De acuerdo al tema de investigación se presentan los resultados de las preguntas de mayor relevancia, relacionadas con los tipos de software y paquetes ofimáticos que utilizan en las instituciones investigadas.

\section{Software de Aplicación.}

Tabla 3.

Identifican ¿Qué es un software?

\begin{tabular}{lcccccc}
\hline Etiqueta & $\begin{array}{c}\text { GAD } \\
\text { Municipal de } \\
\text { Salcedo }\end{array}$ & $\begin{array}{c}\text { Hospital Básico } \\
\text { Yerovi Mackuart de } \\
\text { Salcedo }\end{array}$ & $\begin{array}{c}\text { Cuerpo de } \\
\text { Bomberos de } \\
\text { Pujilí }\end{array}$ & $\begin{array}{c}\text { Cooperativa de Ahorro y } \\
\text { Crédito Occidental de } \\
\text { Pujilí }\end{array}$ & Total & Porcentaje \\
\hline SI & 52 & 32 & 6 & 5 & 95 & $88,79 \%$ \\
NO & 8 & 2 & 2 & 0 & 12 & $11,21 \%$ \\
Total & 60 & 34 & 8 & 5 & 107 & $100,00 \%$
\end{tabular}

Fuente: Autores (2021)

En la Tabla 3, se puede evidenciar que en su gran mayoría los servidores públicos si tienen conocimiento sobre el software y su funcionamiento con un porcentaje predominante del $(88,79)$ y en un porcentaje mínimo de $(11,21)$ desconocen sobre este término. Esto es preocupante ya que en la época actual si los funcionarios no conocen y manejan la tecnología se les considera analfabetos digitales. 


\section{Tabla 4}

Identifican ¿Qué es un Procesador de Texto?

\begin{tabular}{lcccccc}
\hline Etiqueta & $\begin{array}{c}\text { GAD } \\
\text { Municipal de } \\
\text { Salcedo }\end{array}$ & $\begin{array}{c}\text { Hospital Básico } \\
\text { Yerovi Mackuart de } \\
\text { Salcedo }\end{array}$ & $\begin{array}{c}\text { Cuerpo de } \\
\text { Bomberos de } \\
\text { Pujilí }\end{array}$ & $\begin{array}{c}\text { Cooperativa de Ahorro y } \\
\text { crédito Occidental de } \\
\text { Pujilí }\end{array}$ & Total & Porcentaje \\
\hline SI & 56 & 29 & 8 & 5 & 98 & $91,59 \%$ \\
NO & 4 & 5 & 0 & 0 & 9 & $8,41 \%$ \\
Total & 60 & 34 & 8 & 5 & 107 & $100,00 \%$
\end{tabular}

Fuente: Autores (2021)

En la Tabla 4, se puede observar que existe un porcentaje del $(91,59)$ que corresponde aquellos funcionarios que conocen sobre esta aplicación; pero en un mínimo porcentaje de $(8,41)$ no tiene idea de que se trata. El término procesador de texto en los funcionarios no es conocido, pero si se dice Word ahí si lo identifican, por eso es necesario adoptar una cultura digital en las organizaciones mismas que permitan estar familiarizadas con estos términos.

Tabla 5

Qué Tipos de software de oficina utiliza con más frecuencia en el GAD Municipal del cantón Salcedo.

\begin{tabular}{|c|c|c|c|c|c|}
\hline Software & Etiqueta SI & Porcentaje SI & Etiqueta NO & Porcentaje NO & $\begin{array}{c}\text { Total } \\
\text { encuestados }\end{array}$ \\
\hline Word & 58 & $96,67 \%$ & 2 & $3,33 \%$ & 60 \\
\hline Excel & 58 & $96,67 \%$ & 2 & $3,33 \%$ & 60 \\
\hline Power Point & 33 & $55,00 \%$ & 27 & $45,00 \%$ & 60 \\
\hline Adobe Acrobat PDF & 35 & $58,33 \%$ & 25 & $41,67 \%$ & 60 \\
\hline Google Calendar & 13 & $21,67 \%$ & 47 & $78,33 \%$ & 60 \\
\hline
\end{tabular}

Fuente: Autores (2021)

En la Tabla 5 se puede observar que de un total de 8 aplicaciones detalladas en la encuesta se ha seleccionado 5 por tener mayor porcentaje de acogida para su correspondiente análisis, concluyendo que en el GAD del cantón Salcedo las aplicaciones con mayor porcentaje de utilización son Microsoft Word y Excel con $(96,67)$. También los funcionarios de esta institución utilizan para sus actividades diarias otras aplicaciones de oficina como son: Power Point, Adobe Acrobat Professional para abrir documentos con extensión .PDF y Google Calendar para agendar reuniones y eventos importantes en la institución con un porcentaje menor al $(58,34)$.

Tabla 6

Qué Tipos de software de oficina utiliza con más frecuencia en el Hospital Básico Yerovi Mackuart de Salcedo

\begin{tabular}{|c|c|c|c|c|c|}
\hline Software & Etiqueta SI & Porcentaje SI & Etiqueta NO & Porcentaje NO & $\begin{array}{c}\text { Total } \\
\text { encuestados }\end{array}$ \\
\hline Word & 32 & $94,12 \%$ & 2 & $5,88 \%$ & 34 \\
\hline
\end{tabular}

Fuente: Autores (2021) 
Tabla 6

Qué Tipos de software de oficina utiliza con más frecuencia en el Hospital Básico Yerovi Mackuart de Salcedo (continuación)

\begin{tabular}{lccccc}
\hline \multicolumn{1}{c}{ Software } & Etiqueta SI & Porcentaje SI & Etiqueta NO & Porcentaje NO & $\begin{array}{c}\text { Total } \\
\text { encuestados }\end{array}$ \\
\hline Excel & 33 & $97,06 \%$ & 1 & $2,94 \%$ & 34 \\
Power Point & 24 & $70,59 \%$ & 10 & $29,41 \%$ & 34 \\
Adobe Acrobat PDF & 20 & $58,82 \%$ & 14 & $41,18 \%$ & 34 \\
Hoja de cálculo de Google & 15 & $44,12 \%$ & 19 & $55,88 \%$ & $\mathbf{3 4}$ \\
\hline
\end{tabular}

Fuente: Autores (2021)

En la Tabla 6 se puede observar que de un total de 12 aplicaciones detalladas en la encuesta se ha seleccionado 5 por tener mayor porcentaje de acogida para su correspondiente análisis, concluyendo que en el Hospital Básico Yerovi Mackuart del cantón Salcedo las aplicaciones con mayor porcentaje de utilización son Microsoft Word $(94,12)$ y Excel con $(97,06)$. También los funcionarios de esta institución utilizan para sus actividades diarias otras aplicaciones de oficina como son: Power Point, Adobe Acrobat Professional para abrir documentos con extensión.PDF y la hoja de cálculo de Google con un porcentaje menor al $(70,60)$. Esto demuestra el grado de responsabilidad y la preparación en el manejo de aplicaciones de oficina que existe en la institución.

Tabla 7

Qué Tipos de software de oficina utiliza con más frecuencia en la Cooperativa de Ahorro y Crédito Occidental del cantón Pujilí

\begin{tabular}{lccc}
\hline \multicolumn{1}{l}{ Software } & Etiqueta SI & Porcentaje SI & $\begin{array}{c}\text { Total } \\
\text { encuestados }\end{array}$ \\
\hline Word & & & $\mathbf{5}$ \\
Excel & 5 & $100,00 \%$ & $\mathbf{5}$ \\
Power Point & 5 & $100,00 \%$ & $\mathbf{5}$ \\
Google Docs & 5 & $100,00 \%$ & $\mathbf{5}$ \\
Hoja de cálculo de Google & 5 & $100,00 \%$ & $\mathbf{5}$ \\
\hline
\end{tabular}

Fuente: Autores (2021)

En la Tabla 7 se puede observar que de un total de 12 aplicaciones detalladas en la encuesta se ha seleccionado 5 por tener mayor porcentaje de acogida para su correspondiente análisis, concluyendo que en la Cooperativa de Ahorro y Crédito Occidental del cantón Pujilí todas las aplicaciones son conocidas y utilizadas por los funcionarios con un porcentaje de (100). Esto permite demostrar también que la empresa se preocupa por el bienestar de los empleados y el adecuado servicio que se dé al público por este motivo la capacitación es permanente en esta empresa privada. 
Tabla 8

Qué Tipos de software de oficina utiliza con más frecuencia en el Cuerpo de bomberos del cantón Pujilí

\begin{tabular}{lccccc}
\hline \multicolumn{1}{c}{ Software } & Etiqueta SI & Porcentaje SI & Etiqueta NO & Porcentaje NO & $\begin{array}{c}\text { Total } \\
\text { encuestados }\end{array}$ \\
\hline Word & 7 & $87,50 \%$ & 1 & $12,50 \%$ & $\mathbf{8}$ \\
Excel & 7 & $87,50 \%$ & 1 & $12,50 \%$ & $\mathbf{8}$ \\
Recortes de Windows & 7 & $87,50 \%$ & 1 & $12,50 \%$ & $\mathbf{8}$ \\
Power Point & 6 & $75,00 \%$ & 2 & $25,00 \%$ & $\mathbf{8}$ \\
Adobe Acrobat & 5 & $62,50 \%$ & 3 & $37,50 \%$ & $\mathbf{8}$ \\
Professional (PDF) & & & & &
\end{tabular}

Fuente: Autores (2021)

En la Tabla 8 se puede observar que de un total de 12 aplicaciones detalladas en la encuesta se ha seleccionado 5 por tener mayor porcentaje de acogida para su correspondiente análisis, concluyendo que en el Cuerpo de bomberos del cantón Pujilí las aplicaciones con mayor porcentaje de utilización son: Recortes de Windows, Microsoft Word y Excel con $(87,50)$. También los funcionarios de esta institución utilizan para sus actividades diarias otras aplicaciones de oficina como son: Power Point, Adobe Acrobat Professional para abrir documentos con extensión .PDF con un porcentaje menor o igual al (75). Esto demuestra el grado de responsabilidad y la preparación en el manejo de aplicaciones de oficina que existe en la institución.

\section{Conclusiones}

- En la actualidad las actividades gerenciales y el manejo de información en el mundo entero a sufrido un cambio profundo por el COVID-19, muchos empresarios han tenido que cerrar sus negocios, despedir empleados y los que han logrado sobrevivir a esta crisis económicamente mundial necesitan de una tecnología adecuada para poder cumplir con los procesos y estar conectados con los cliente; es por ello que en las instituciones públicas y privadas encuestadas en su gran mayoría están realizando teletrabajo y para poder cumplir con las actividades laborales están utilizando paquetes ofimáticos, internet entre otros.

- Se identifica al personal femenino como eje predominante en las instituciones encuestadas, esto se debe a que en su gran mayoría estos servidores públicos cumplen con actividades de gestión documental, manejo de información física y digital, archivística y atención al cliente. Sin desmerecer la capacidad intelectual al personal masculino de esta manera se está evidenciando que las mujeres están mejor preparadas para ocupar estos cargos y manejar los procesos administrativos, gestión documental en la empresa.

- En la época actual, la utilización del software se ha convertido en una herramienta importante en las instituciones públicas y privadas, porque por medio de ello estas instituciones se están comunicando con los clientes, redactando documentos administrativos, realizando presentaciones para las reuniones de trabajo, 
agendando reuniones, almacenando información digital en la nube, entre otras cosas. En este trabajo investigativo se logró determinar que el $88,79 \%$ de las personas entrevistadas conocen este término y algunos servidores públicos no identifican la palabra software.

- Pocos son los servidores públicos que no identifican un procesador de texto, pero si se les menciona Microsoft Word, todos lo entienden. Cabe mencionar que no solamente Word es considerado como un procesador de texto también un procesador de texto es: Google docs, open office writer, Abi Word, Word Pad, entre otros. Microsoft Word es el procesador de texto utilizado en estas instituciones con más del 87,50\% de aceptación, los trabajos que realizan con este software es la redacción de los documentos administrativos, elaboración de proyectos, entre otras cosas.

- Haciendo un análisis comparativo en las cuatro instituciones investigadas se puede deducir que las aplicaciones de oficina más utilizadas son Microsoft Word y Excel. Estas dos aplicaciones se han convertido como referente en las 4 instituciones por sus múltiples beneficios, ventajas y fácil de usar. Con estas aplicaciones de oficina se llevan a cabo los procesos administrativos, gestión documental y la elaboración de información física y digital.

- Es necesario que la Universidad Técnica de Cotopaxi brinde capacitaciones permanentes a los servidores públicos no solamente de estas instituciones investigadas sino también proponer capacitaciones abiertas a todo público a través de la dirección de educación continua de la UTC sobre el uso de aplicaciones de oficina como son: Office 365, aplicaciones de Google, Open Office, Libre Office, Microsoft Office, entre otros.

\section{Referencias bibliográficas:}

Sánchez, E. (2008). Las tecnologías de información y comunicación (tic) desde una perspectiva social. Revista Educare Vol. XII, N Extraordinario, 155-162. [En línea]. Recuperado de: https://www.redalyc.org/pdf/1941/194114584020.pdf

Cano, G. (2018). Las TICs en las empresas evolución de la tecnología y cambio estructural en las organizaciones. Dominio de las Ciencias, Vol. 4, No. 1, págs. 499-510. [En línea]. Recuperado de:

https://dialnet.unirioja.es/servlet/articulo?codigo $=6313252$

Yi, Y., Lagniton, P., Ye, S., Li, E., y Xu, R. H. (2020). covid-19: what has been learned and to be learned about the novel coronavirus disease [covid-19: lo que se ha aprendido y lo que se debe aprender sobre la nueva enfermedad del coronavirus]. International Journal of Biological Sciences, 16, 10, 1753-1766. https:// doi.org/10.7150/ijbs.45134. 
Sanz, I., Sáinz González, J., Capilla, A. (2020). Efectos de la Crisis del Coronavirus en la Educación Superior. Organización de Estados Iberoamericanos para la Educación, la Ciencia y la Cultura (oei). https://oei.org.br/arquivos/informe-covid-19d.pdf

Martín, C. (2019). Las TIC en la Administración Pública. Blog de la Universidad de Salamanca. [En línea]. Recuperado de:

https://diarium.usal.es/crisgggmartin/2019/04/22/las-tic-en-la-administracionpublica/

Valverde, J. (2007). El software libre y las buenas prácticas educativas con TIC. Comunicación y Pedagogía, 222, 48-55. [En línea]. Recuperado de: https://n9.cl/pcm2e

Arriola, O. Tecuatl, G \& González, G. (2011). Software propietario vs software libre: una evaluación de sistemas integrales para la automatización de bibliotecas. Investigación bibliotecológica, vol. 25(54), 37-70. [En línea]. Recuperado de: http://www.scielo.org.mx/scielo.php?script=sci_arttext\&pid=S0187358X2011000200003

Ávila, O. (2011). Computaci’on en la nube. ContactoS 80, 45-52 [En línea]. Recuperado de: https://n9.cl/5oyu

Hernández, C \& Carpio, N. (2019). Introducción a los tipos de muestreo. Revista ALERTA, Vol. $2 \mathrm{~N}^{\circ}$ 1. [En Línea] Recuperado de: https://alerta.salud.gob.sv/wpcontent/uploads/2019/04/Revista-ALERTA-An\%CC\%83o-2019-Vol.-2-N-1-vf75-79.pdf

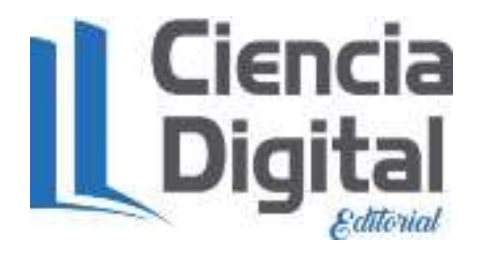




\section{PARA CITAR EL ARTÍCULO INDEXADO.}

Flores Lagla, G. A., Jácome Segovia, D. F., Paucar Coque, L. M., \& Borja Brazales, Y. P. (2021). Herramientas ofimáticas aplicadas en los procesos administrativos en las instituciones del cantón Salcedo y Pujilí . ConcienciaDigital, 4(3.2), 66-78. https://doi.org/10.33262/concienciadigital.v4i3.2.1844

\section{Ligital}

El artículo que se publica es de exclusiva responsabilidad de los autores y no necesariamente reflejan el pensamiento de la Revista Conciencia Digital.

El artículo queda en propiedad de la revista y, por tanto, su publicación parcial y/o total en otro medio tiene que ser autorizado por el director de la Revista Conciencia Digital.

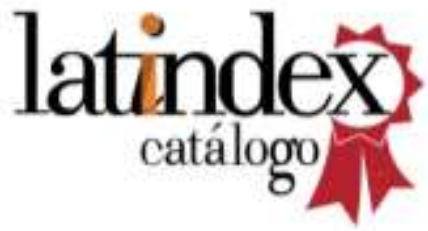

